\title{
Un récepteur du glycocolle responsable d'une maladie neurologique, I'hyperekplexie
}

L'hyperekplexie héréditaire, ou maladie des sursauts (startle disease), est une affection rare, autosomique dominante, marquée par une hypertonie chez le nourrisson et une persistance de réactions exagérées aux sursauts. Cette hypertonie figure parmi les causes de mort subite. Un retard moteur important se rattrape lors de la croissance, ne laissant que des contractions inopinées après stimulation, auditive surtout. Ces réactions sont atténuées par la benzodiazépine clonazepam, qui active l'action du GABA sur la neurotransmission. Or deux récepteurs du GABA, ainsi qu'un récepteur du glutamate et un récepteur $\alpha$ adrénergique ont tous leur gène localisé sur le bras long du chromosome 5 . L'attention a donc été attirée sur ce chromosome; pourtant, les études fines de liaison ont montré, dans plusieurs familles d'hyperekplexie, que le locus de la maladie était bien sur le 5 mais en dehors de ceux des récepteurs cités [1]. Récemment [2], le gène de l'hyperekplexie et celui de la sous-unité $\alpha_{1}$ du récepteur du glycocolle (GLRA1) furent localisés dans la partie distale du bras long du chromosome 5. Le marqueur le plus proche de GLRAl, appelé D5S119, ne présente aucune recombinaison avec le locus de l'hyperekplexie dans aucune famille de malades [2]. GLRAl apparaîtrait comme un gène candidat d'autant meilleur que le récepteur du glycocolle reconnaît comme antagoniste la strychnine, qui provoque chez la souris de l'hypertonie et une exagération de la réponse au sursaut. Une équipe réunissant des chercheurs de Californie, du Texas et de l'Ontario a donc décidé d'explorer cette voie [3].

unité $\alpha$ du récepteur, qui mesure $1,7 \mathrm{~kb}$, est connue depuis 1990 [4]. La protéine déduite compte 452 acides aminés. A partir de cet ADNc, on a préparé des cosmides qui ont permis d'analyser la structure du gène. Celui-ci s'étend sur au moins $150 \mathrm{~kb}$, et contient 8 exons, dont le premier est non codant. L'analyse de la séquence protéique suggère la présence de quatre segments transmembranaires, avec des extrémités et une boucle extracellulaires et deux boucles intracellulaires (figure 1).

Les exons furent analysés pour la recherche d'anomalies, d'abord par la méthode d'électrophorèse sur gel en gradient dénaturant. Parmi les exons examinés dans sept familles, seul l'exon 6 montra des particularités: deux variants non retrouvés chez les témoins furent observés, l'un dans une seule famille, l'autre dans trois. La séparation des allèles dans des hybrides somatiques homme-hamster montra que, chez chaque malade, on trouvait un allèle normal et un allèle muté. Le séquençage de l'exon 6 montra une mutation unique dans chaque cas, siégeant au niveau du même nucléotide 1192, faisant partie du codon arginine en position 271. Dans une famille, une transversion $G \rightarrow T$ convertissait l'arginine en leucine, dans trois familles une transition $G$ $\rightarrow$ A la changeait en glutamine; dans les deux cas donc, un acide aminé basique était remplacé par un neutre. Dans les trois dernières familles, l'exon 6 ne paraissait pas en cause, et une exploration du reste de la molécule est en cours. D'après les auteurs, l'identification de ces mutations procure le premier exemple d'une maladie génétique humaine due à une lésion molécu- laire affectant directement un récepteur de neurotransmetteur dans le système nerveux central [3].

Reste à comprendre comment une lésion d'une sous-unité de ce récepteur peut provoquer hypertonie et réaction de sursaut. Le récepteur du glycocolle est un canal chlore hétéro-oligomérique, doté d'une grande diversité de sous-types. Il existe à la fois des isoformes de la chaîne $\alpha$, codées par des gènes différents, et des variants intragéniques par épissage alternatif. L'isoforme en cause dans la maladie est formée de deux types de sous-unités, une sous-unité $\alpha$ de $48 \mathrm{kDa}$, une sousunité $\beta$ de $58 \mathrm{kDa} ; 3 \alpha$ et $2 \beta$ s'unissent en une structure pentamérique [5]. Si la présence d'une seule sous-unité $\alpha$ mutée suffit à empêcher le canal d'être fonctionnel, on peut calculer qu'un hétérozygote ne porterait que $12,5 \%$ de canaux fonctionnellement normaux, expliquant le caractère génétique dominant.

La topologie transmembranaire est analogue à celle des récepteurs du GABA, de l'ACh, et du récepteur nicotinique. L'arginine en position 271 siège dans la courte boucle extracellulaire entre M2 et M3, et est probablement importante pour la liaison du chlore ou son entrée dans le canal.

Il est enfin probable qu'il existe, comme dans nombre d'affections neurologiques, un ou plusieurs modèles animaux de déficit en récepteur du glycocolle. On en connaît deux gènes responsables possibles. Le mutant spasmodique spd a son gène sur le chromosome 11 de la souris, dans une région homologue de q22-q32 du 5 humain [6]. Certes, la maladie murine est récessive, mais on sait bien maintenant 


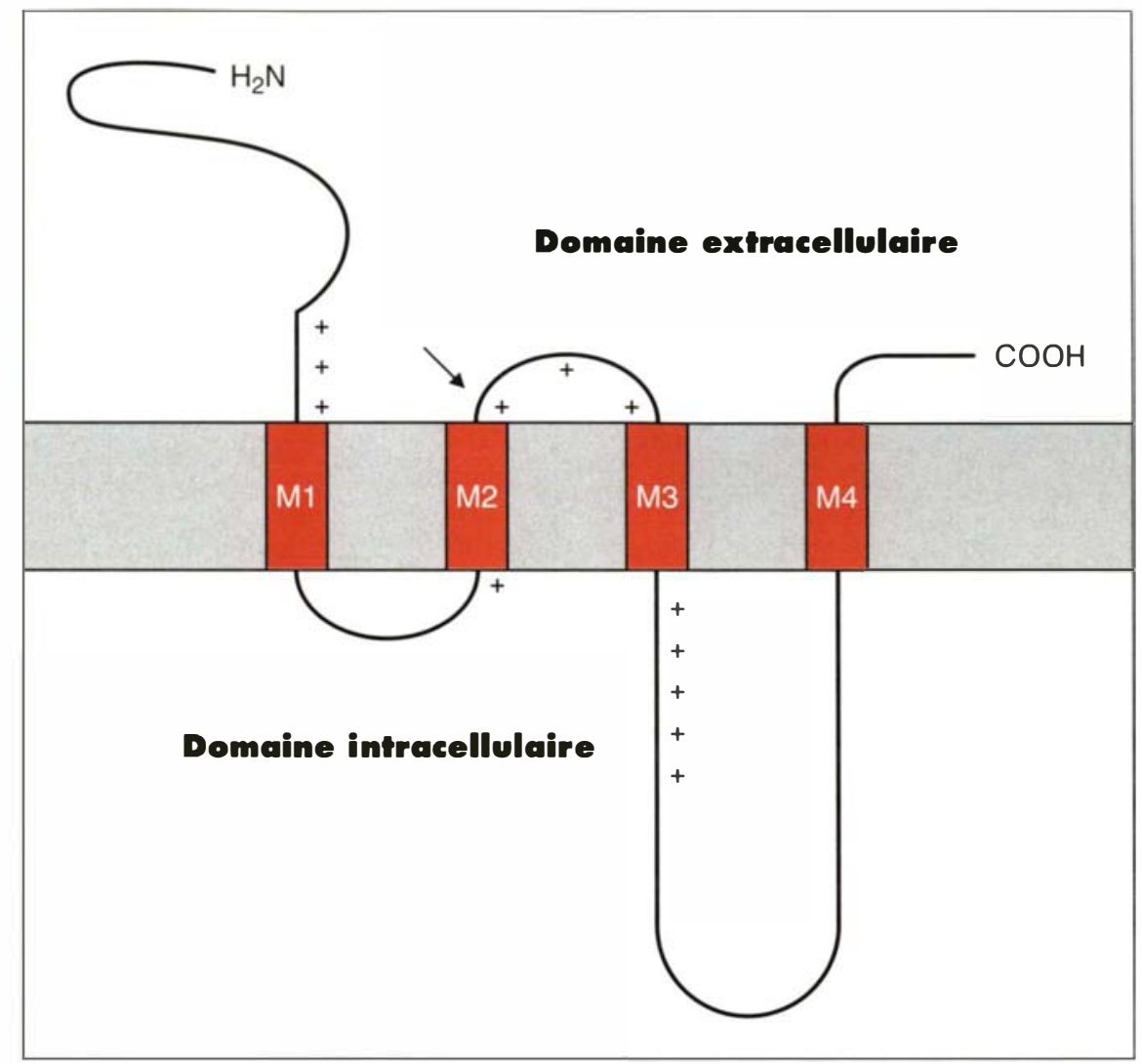

Figure 1. Structure de la sous-unité $\alpha$ du récepteur du glycocolle. Les quatre domaines transmembranaires sont numérotés MI-M4. + : régions chargées positivement. La position de l'arginine 271 mutée est indiquée par une flèche. (D'après [3].)

que l'atteinte du même gène peut donner des affections, soit dominantes, soit récessives. Quant au mutant spastique (spa), son gène siège sur le chromosome 3 qui n'est pas homologue du 5 humain. Il pourrait s'agir là d'une mutation d'une autre sousunité du récepteur.

J.C.D.

1. Ryan SG, Dixon MJ, Nigro MA, Kelts A, Markand OM, Terry JC, Shiang R, Wasmuth IJ, O'Connell P. Genetic and radiation hybrid mapping of the hyperekplexia region on chromosome 5q. Am J Hum Genet 1992; 51 : 1334-43.

$\mathrm{m} / \mathrm{s} n^{\circ} 3$ vol. 10 , mars 94
2. Warrington JA, Benctsson U, Bailey SK, Lovett M, Wasmuth IJ. A comparison of three methods to produce a high resolution physical map of 11 genes on the distal region of the long arm of human chromosome 5. Am J Hum Genet 1992; 51 : A248 (suppl).

3. Shiang R, Ryan SG, Zhu YZ, Hahn AF, O'Connell P, Wasmuth IJ. Mutations in the $\alpha$ subunit of the inhibitory glycine receptor cause the dominant neurologic disorder, hyperekplexia. Nature Genet 1993; 5 : 351-8. 4. Genningloh G, Schmieden V, Schofield PR, Seeburg PH, Siddique T, Mohandas TK, Becker CM, Betz H. Alpha subunit variants of the human glycine receptor. Primary structures, functional expression and chromosomal localization of the corresponding genes. EMBO J 1990; 9: 771-6.

5. Buckwalter MS, Testa CM, Noebels JM, Camper SA. Genetic mapping and evaluation of candidate genes for spasmodic, a neurological mouse mutation with abnormal startle response. Genomics 1993; 17: 279-85. 